\title{
USO DE ANALOGÍAS ADECUADAS COMO RECURSO DIDÁCTICO PARA LA COMPRENSIÓN DE LOS FENÓMENOS ELECTROQUÍMICOS EN EL NIVEL UNIVERSITARIO INICIAL
}

\author{
VASINI, ENRIQUE JULIO y DONATI, EDGARDO RUBÉN \\ Departamento de Química. Facultad de Ciencias Exactas \\ Universidad Nacional de La Plata, 47 y 115 (1900) La Plata. Argentina
}

\begin{abstract}
SUMMARY
In this paper, we propose a complementary strategy to introduce electrochemical cells in introductory courses of chemistry. Frequently some of the concepts related to electrochemical processes, such as electric work, reversible and irreversible changes, maximum work and differences and similarities between galvanic and electrolytic cells among others, are poorly developed in this kind of courses. We propose, for the introductory treatment of these concepts, an analogy between isothermal expansion of a perfect gas and an electrochemical process. The analogy is qualitatively and quantitatively discussed.
\end{abstract}

\section{INTRODUCCIÓN}

La incorporación de nuevos conceptos requiere de la activación de ideas o esquemas mentales sobre los cuales pueda asentarse la nueva información. La función de una analogía adecuada es facilitar el aprendizaje, y su importancia es tanto mayor cuando menores sean, en los alumnos, las ideas previas existentes respecto de un dominio de conocimientos determinado.

Una vez que se ha alcanzado cierto nivel cognitivo sobre un tema determinado, se generan, conscientemente o no, analogías y relaciones con conocimientos previos. La introducción por parte del docente de una analogía conceptual y estructuralmente adecuada al problema, fácilmente generalizable y con referencia a temas previos del mismo curso (Donati y Andrade Gamboa, 1990; Pozo, Sanz, Gómez Crespo y Limón, 1991; Pozo y Gómez Crespo, 1998) puede suplantar, enriquecer o guiar las analogías que el alumno inevitablemente generará y, probablemente, el resultado será una mejor compresión del tema. De todos modos, la efectividad de la analogía en la enseñanza depende de gran número de factores entre los cuales se encuentran el tipo de analogía, el grupo de alumnos en los cuales se aplica, el tiempo dedicado a la explicación de la analogía, etc. (Friedel, Gabel y Samuel, 1990).

Muchos estudiantes y docentes de química coinciden en las dificultades asociadas al aprendizaje del tema de electroquímica. Esto ha generado un elevado número de investigaciones que muestran incorrectas interpretaciones o errores en la comprensión de conceptos de este tema en estudiantes de distintos niveles de la enseñanza (Garnett y Treagust, 1992, 1992b; Senger y Greenbowe, 1997). En estos trabajos se describen con detalle las 
dificultades asociadas con las propias reacciones de óxido-reducción, con los responsables del transporte de la corriente eléctrica a través de la solución y del puente salino o con la interpretación de las cargas asignadas a cada electrodo.

Los problemas mencionados en el párrafo anterior han sido detectados frecuentemente en nuestros cursos junto a otros relacionados con conceptos más complejos que también están incluidos en la currículos de estos cursos. Entre estos problemas podemos mencionar específicamente las dificultades para identificar el marco conceptual y físico común entre celdas electrolíticas y celdas galvánicas y los conceptos de reversibilidad y trabajo eléctrico. Aunque no existe una única causa, es posible identificar algunos factores que inciden en forma directa en la aparición de estos problemas. En el caso particular de una visión no integrada entre ambos tipos de celdas electroquímicas, es probable que la sensación de los alumnos sobre que los procesos que tienen lugar en celdas galvánicas y electrolíticas tienen escasa vinculación está generada porque estos temas suelen desarrollarse en forma separada e independiente. Respecto de los conceptos de reversibilidad y trabajo, probablemente su mayor grado de abstracción y la existencia de otros procesos en los cuales ambos conceptos termodinámicos se pueden tratar de un modo más intuitivo provocan un tratamiento incompleto y, en muchas ocasiones, erróneo en libros de texto y en las clases.

Debido a estos problemas en alcanzar un grado más profundo de comprensión de los conceptos electroquímicos, se han propuesto diversos métodos para la enseñanza de este tema aunque ninguno ha probado ser especialmente efectivo (Senger y Greenbowe, 1997).

En este trabajo, sugerimos el uso de una metodología alternativa para el tratamiento de los procesos de electrodo (pilas y celdas electrolíticas) a nivel universitario inicial. En ésta se utilizan analogías con los procesos de expansión-compresión, reversibles o no, de los gases ideales. Frecuentemente, el uso de las analogías en la enseñanza pretende que el alumno relacione la «química» a situaciones familiares; sin embargo, en este caso utilizamos una analogía también situada en el conocimiento científico aunque basada en conceptos más intuitivos y sencillos y que, habitualmente, está ubicada mucho antes en la currículos que el tema de electroquímica.

\section{LOS PROCESOS DE ELECTRODO Y LA REVERSIBILIDAD TERMODINAMICA}

En la mayoría de los libros de química básica se introduce el concepto de reversibilidad termodinámica utilizando como ejemplo la expansión isotérmica reversible de un gas ideal (Whitten, Gailey y Davis, 1992; Atkins y Jones, 1997; Brown, LeMay y Bursten, 1998; Chang, 1998). Sin duda este sistema se elige debido a su sencillez y a la posibilidad de reconocer en él máquinas e instrumentos de uso común. Esto hace más sencillo desarrollar la imagen mental del sistema ideal transformándose en estado de cuasi equilibrio.

Esta imagen mental se suele evocar convenientemente al representar el sistema como un cilindro con un pistón móvil y reemplazar la presión externa por la presión ejercida por un peso total inicial, fraccionado en pesas iguales (Fig. 1). La presión puede cambiarse en forma discreta retirando o agregando una pesa por vez (deslizándola hacia, o desde, una bandeja adyacente).

El aumento del número de fracciones en las que se divide el peso inicial (es decir, la disminución de cada paso discreto) cambia el valor del trabajo efectuado en una transformación entre iguales estados iniciales y finales. Este cambio puede calcularse y representarse en un diagrama $\mathrm{P}-\mathrm{V}$.

Este ejercicio permite desarrollar con claridad el concepto de trabajo máximo y asociarlo con los conceptos de cuasi equilibrio y de diferencia infinitesimal entre la presión externa y la interna.

En los textos para el nivel universitario inicial, se da por establecido el concepto de reversibilidad termodinámica una vez desarrollado, del modo anterior o de una manera semejante, el tema de la termodinámica de la expansión (compresión) reversible e isotérmica de un gas ideal. El concepto de reversibilidad termodinámica se usa en otros temas del curso, en particular en el tratamiento de pilas y celdas electroquímicas, sin una nueva y mayor revisión.

Figura 1

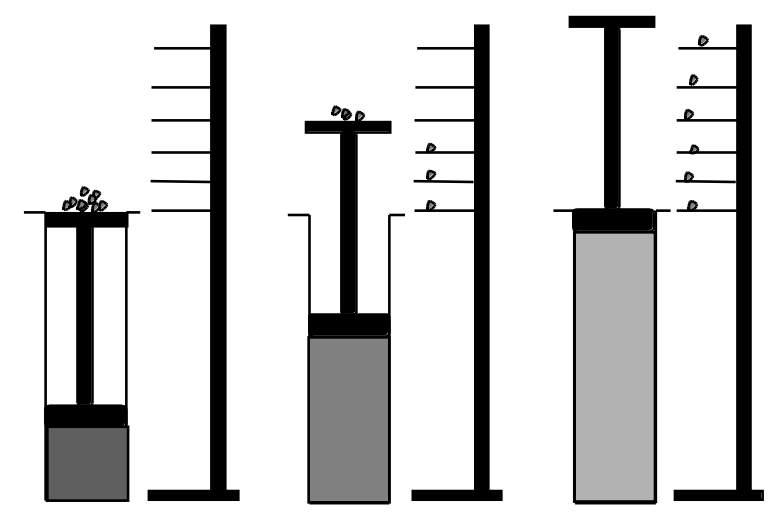

Sin embargo, la idea de reversibilidad termodinámica es suficientemente útil, contraintuitiva y compleja como para merecer un mayor desarrollo. No sólo la presentación elemental de las celdas electroquímicas ofrece un ejemplo ideal para profundizar en este concepto, sino que su revisión ayuda a la comprensión de la electroquímica y elimina separaciones conceptuales artificiales entre pilas y celdas electrolíticas. 


\section{DESARROLLO DE LA ANALOGÍA DIDÁC- TICA PROPUESTA}

En muchos textos de nivel inicial como los indicados en las referencias bibliográficas, el tema de los procesos de electrodo se introduce a través del siguiente ordenamiento:

1) Obtención de electricidad a partir de la reacción química redox.

2) Relación de las reacciones redox con la construcción física de una pila, circuito externo.

3) Concepto de trabajo eléctrico. Cálculo del mismo.

4)Fuerza electromotriz de pilas.

5) Celdas electrolíticas.

El tema 5 suele también tratarse en primer lugar, luego de una introducción a la conducción eléctrica por medio de soluciones electrolíticas. Esta diferencia no afecta nuestra propuesta.

En los textos suele introducirse el concepto de fuerza electromotriz en forma errónea, definiéndola simplemente como «la diferencia de potencial entre los dos electrodos de una celda galvánica» (Brown, LeMay y Bursten, 1998; Chang, 1998). En algunos de estos libros, se informa a continuación, sin justificación, que debe medirse la diferencia de potencial entre los electrodos en condiciones en las que no circule corriente.

Figura 2

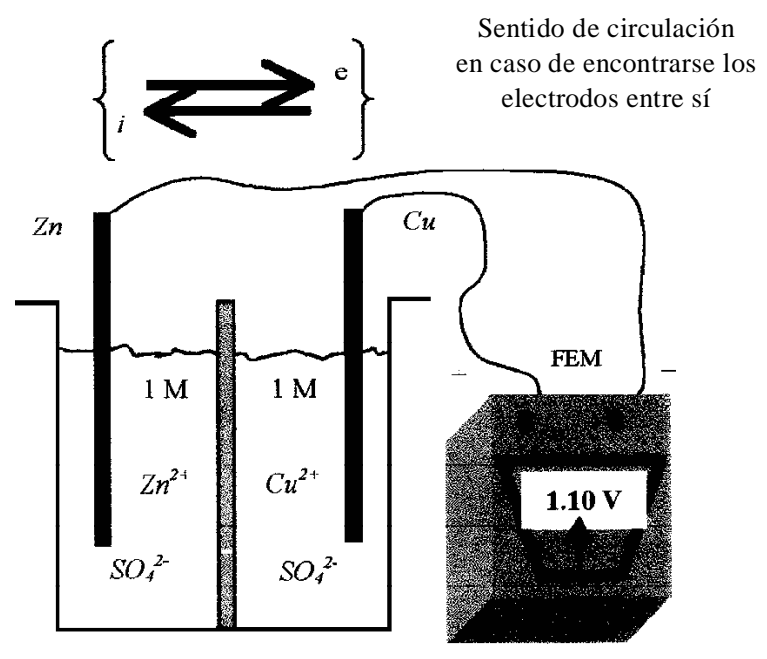

En el centro de la diferencia entre la «fuerza electromotriz de la pila $(\mathrm{FEM}) »$ y la «diferencia de potencial entre los electrodos $(\Delta \mathrm{E}) \gg$ está el concepto de reversibilidad termodinámica. La expresa clarificación de la diferencia entre FEM y $\Delta \mathrm{E}$ puede usarse para reforzar el concepto central de reversibilidad termodinámica y para lograr una comprensión inicial cualitativamente superior de las celdas electroquímicas.

Para ello proponemos la discusión del tema del trabajo eléctrico a través de una analogía con el trabajo de expansión (compresión) isotérmica de un gas ideal.

\section{DESARROLLO DE LA PROPUESTA}

Una vez discutidos los puntos 1 y 2 indicados más arriba, los alumnos habrán comprendido el significado de todos los elementos usados en el esquema simbólico de una pila de Daniell, tal como la de la figura 2. El valor de la diferencia de potencial puede presentarse como un resultado experimental o, mejor aún, puede medirse ante la clase con un multímetro sencillo. Se denominará a esta diferencial de potencial fuerza electromotriz (FEM) de la pila y se indicará que su definición y significado físico quedarán claros en el desarrollo de la clase.

Llegado este punto, se analizarán con los estudiantes los procesos que ocurrirán en la pila si se conecta ésta a un aparato denominado potenciostato (no es necesario ni conveniente detallar el uso y las características del mismo), que permita variar en forma continua la diferencia de potencial entre sus bornes y medir la intensidad de la corriente que circula (Fig. 3).

Figura 3

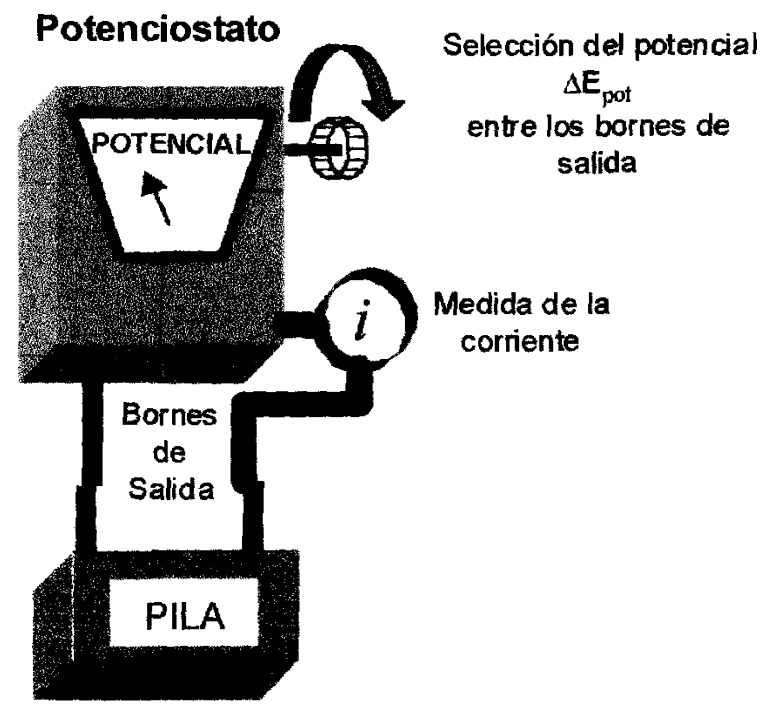

El resultado del análisis de cada caso se facilita si se propone a los alumnos pensar en que sentido y con que intensidad cualitativa circularía la corriente eléctrica 
por una resistencia, si ésta se conectara: $a$ ) al potenciostato, $b$ ) a la pila, y, por último, si se conectaran entre sí, c) la pila y el potenciostato.

Es importante que, durante esta discusión, los alumnos comprendan las relaciones existentes entre la intensidad de la corriente eléctrica y su sentido de circulación, y la velocidad y el sentido de las reacciones redox en la pila.

El resultado de esta discusión podrá resumirse de la manera indicada en el esquema de la figura 4a. Éste deberá compararse, de acuerdo a nuestra propuesta, con el esquema de la figura $4 \mathrm{~b}$. La definición y el significado práctico (termodinámico) de la FEM de la pila, así como la necesidad de su medida en condiciones de intensidad de corriente eléctrica (prácticamente) nula quedarán aclarados y reforzados por su comparación con los procesos de expansión o compresión reversibles o no de un gas ideal.

En las figuras anteriores, puede observarse la relación directa entre conceptos de la analogía y del hecho a explicar: la «presión externa» aplicada, la «presión interna» del gas, el proceso de compresión y el de expansión (en la analogía) pueden asociarse en forma directa a la «diferencia de potencial entre los bornes», la «FEM» de la pila, el funcionamiento de una celda electrolítica y el de una galvánica. A partir de estas relaciones, puede generarse fácilmente una imagen intuitiva de los procesos que pueden ocurrir en una celda electroquímica.

En el trabajo eléctrico producido por la pila ( $\left.\mathrm{w}_{\text {elect }}\right)$, el factor extensivo es la carga eléctrica transportada $(q)$ y el intensivo es la diferencia de potencial entre los electrodos $\left(\Delta \mathrm{E}_{\text {pila }}\right)$, es decir:

$$
\mathrm{W}_{\text {elect }}=\int \Delta \mathrm{Exdq}
$$

No es fácil obtener una representación mental convincente y clara de la diferencia de potencial entre los electrodos como factor intensivo del trabajo eléctrico. La analogía con la expansión isotérmica reversible resulta nuevamente útil.

En la expansión isotérmica reversible de un gas ideal, el trabajo máximo de expansión realizado por el sistema se obtiene cuando cada cambio de volumen (factor extensivo) se realiza contra una presión externa (factor intensivo) infinitesimalmente menor que la interna. Es decir, el trabajo realizado en una etapa infinitesimal es: $\delta \mathrm{w}_{\text {exp. }}=\mathrm{P}_{\text {ext }} \mathrm{XdV}$ con $\mathrm{P}_{\text {ext }}=\mathrm{P}_{\text {int }}-\mathrm{dP}$. Resulta obvio que la presión externa y, por lo tanto, el trabajo no pueden ser mayores, ya que, si $\mathrm{P}_{\mathrm{ext}}=\mathrm{P}_{\mathrm{int}}$, no hay transformación $\mathrm{y}$, si $\mathrm{P}_{\text {ext }}>\mathrm{P}_{\text {int }}$, ocurre una compresión (se entrega trabajo al sistema) y no una expansión.

De idéntica manera, el trabajo eléctrico máximo realizado por una pila resulta cuando cada carga se transfiere contra la máxima diferencia de potencial entre los electrodos, cosa que ocurre cuando hay una diferencia infinitesimal entre la diferencia de potencial en el potenciostato y la fuerza electromotriz de la pila. O sea, el trabajo realizado por la pila en una etapa infinitesimal sería $\delta w_{\text {elect. }}=\Delta E_{\text {pila }} X d q$ con $\Delta E_{\text {pila }}=F E M_{\text {pila }}-d E$. Si el $\Delta E$ es mayor y supera a la $\mathrm{FEM}_{\text {pila, }}$ se entregará trabajo eléctrico a la pila y la celda funcionará como celda electrolítica (ocurrirá una electrólisis) y no como una celda galvánica.

Así, la analogía no sólo refuerza el concepto de reversibilidad termodinámica, sino que nuevamente permite ver la similitud entre «diferencia de potencial entre los bornes» de la pila y «presión externa» de un sistema gas ideal-cilindro-pistón móvil y entre la «FEM»y «presión interna» respectivamente, para los mismos sistemas. Además se analizan celdas electrolíticas y galvánicas como «las dos caras de una misma moneda».

\section{DESARRQLLO CUANTITATIVO DE LA ANALOGIA}

La analogía del trabajo eléctrico con el trabajo de expansión puede desarrollarse cuantitativamente por medio del cálculo del trabajo eléctrico total en una pila sencilla del tipo $\mathrm{M}_{1}\left|\mathrm{M}_{1}{ }^{\mathrm{n}+}\left(\mathrm{C}_{\mathrm{M} 1}\right)\right|\left|\mathrm{M}_{2}{ }^{\mathrm{m}+}\left(\mathrm{C}_{\mathrm{M} 2}\right)\right| \mathrm{M}_{2}$, tal como la pila de Daniell, cuando éste se efectúa contra una diferencia de potencial infinitesimalmente menor que la de la pila (reversiblemente), o por medio de varias etapas discretas.

Por ejemplo, la FEM de una pila de Daniell, con concentraciones iniciales de $\mathrm{Zn}^{2+}$ y de $\mathrm{Cu}^{2+}$ iguales a 1 Molar es 1,1 volts. Supongamos que los volúmenes de catolito y anolito son ambos iguales a 1 litro, de modo que hay inicialmente tanto un mol de $\mathrm{Zn}^{2+}$ como de $\mathrm{Cu}^{2+}$. Se puede descargar esta pila hasta que la concentración de $\mathrm{Zn}^{2+}$ aumente a $1,9 \mathrm{M}$ y la de $\mathrm{Cu}^{2+}$ decrezca hasta $0,1 \mathrm{M}$, por ejemplo, en tres etapas discretas:

- Etapa 1: Transportar la carga correspondiente a la conversión de 0,3 moles contra un potencial igual a la FEM de la pila $\mathrm{Zn}\left|\mathrm{Zn}^{2+}(1,3 \mathrm{M}) \| \mathrm{Cu}^{2+}(0,7 \mathrm{M})\right| \mathrm{Cu}$; es decir, contra un potencial final igual a la FEM de la pila luego de la tranferencia de carga.

- Etapa 2: Desde un estado inicial igual al estado final del proceso $a$ ), transportar la carga correspondiente a la conversión de 0,3 moles contra un potencial igual a la FEM de la pila $\mathrm{Zn}\left|\mathrm{Zn}^{2+}(1,6 \mathrm{M}) \| \mathrm{Cu}^{2+}(0,4 \mathrm{M})\right| \mathrm{Cu}$.

- Etapa 3: Desde un estado inicial igual al estado final del proceso $b$ ) transportar la carga correspondiente a la conversión de 0,3 moles contra un potencial igual a la FEM de la pila $\mathrm{Zn}\left|\mathrm{Zn}^{2+}(1,9 \mathrm{M}) \| \mathrm{Cu}^{2+}(0,1 \mathrm{M})\right| \mathrm{Cu}$.

El proceso se resume en la tabla I.

El trabajo realizado puede observarse y calcularse como el área bajo la función $E=f(q)$. En la figura 5 se muestran curvas calculadas para los mismos estados inicial y final, con la conversión realizada en distinto número de pasos: 3 (línea gris de puntos), 9 (línea gris de rayas) y 90 (prácticamente equivalente a infinitos pasos, línea continua). 
Figuras $4 \mathrm{a}$ y $4 \mathrm{~b}$

Trabajo máximo
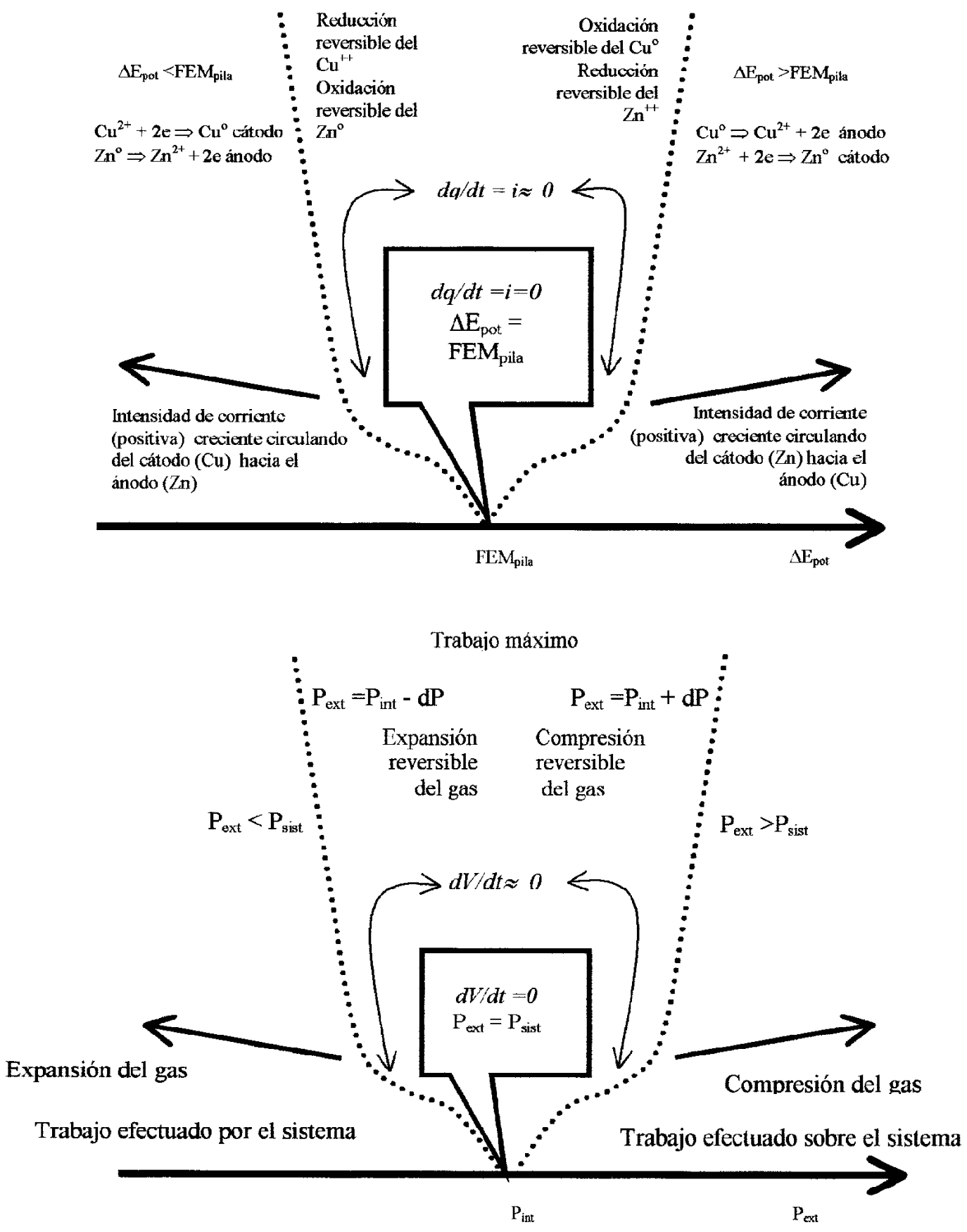
Tabla I

\begin{tabular}{|c|c|c|c|c|c|}
\hline Etapa & $\begin{array}{c}\text { Moles } \\
\text { totales } \\
\text { convertidos }\end{array}$ & $\begin{array}{c}\mathbf{E}=\text { Potencial } \\
\text { externo } \\
\text { (volts) }\end{array}$ & $\begin{array}{c}\mathbf{Q}= \\
\text { Carga total } \\
\text { transportada } \\
\text { (coulombs) }\end{array}$ & $\begin{array}{c}\mathbf{q}= \\
\text { Carga parcial } \\
\text { transportada } \\
\text { (coulombs) }\end{array}$ & $\begin{array}{c}\mathbf{W}=\mathbf{E}, \mathbf{q} \\
\text { Trabajo eléctrico } \\
\text { (joules) }\end{array}$ \\
\hline 1 & 0,000 & 1,100 & 0,000 & 0,000 & 0,000 \\
\hline 2 & 0,300 & 1,092 & 57,900 & 57,900 & 63,231 \\
\hline 3 & 0,600 & 1,082 & 115,800 & 57,900 & 62,663 \\
\hline
\end{tabular}

El potencial correspondiente a cada punto, la carga total transportada y el trabajo total pueden calcularse convenientemente usando un programa de «hoja de cálculo», tal como el Excel de Microsoft

Los trabajos totales calculados son: para 3 etapas, 187,4 $\mathrm{kJ}$; para 9 etapas, $188,2 \mathrm{~kJ}$; y para 90 etapas, $188,6 \mathrm{~kJ}$ respectivamente. Este último coincide, dentro de las cuatro cifras significativas, con el trabajo reversible exacto, calculado por medio de:

$$
\int_{q_{x=0}}^{q_{x=0.9}} \Delta E(q) \times d q
$$

En base a lo desarrollado, el docente podrá hacer uso de la analogía en forma reiterada, para establecer una imagen intuitiva, cualitativa o incluso cuantitativa de los procesos que pueden tener lugar en una celda electroquímica.

La presente analogía no ha sido probada aún en forma sistemática en función de observar sus potenciales ventajas respecto de otro tipo de estrategia para la enseñanza de electroquímica. Sin embargo, los autores han utilizado esta estrategia de introducir el tema en forma parcial o total en los cursos a su cargo en los últimos años. Esto les ha permitido comprobar un incremento significativo en la comprensión de temas frecuentemente signados

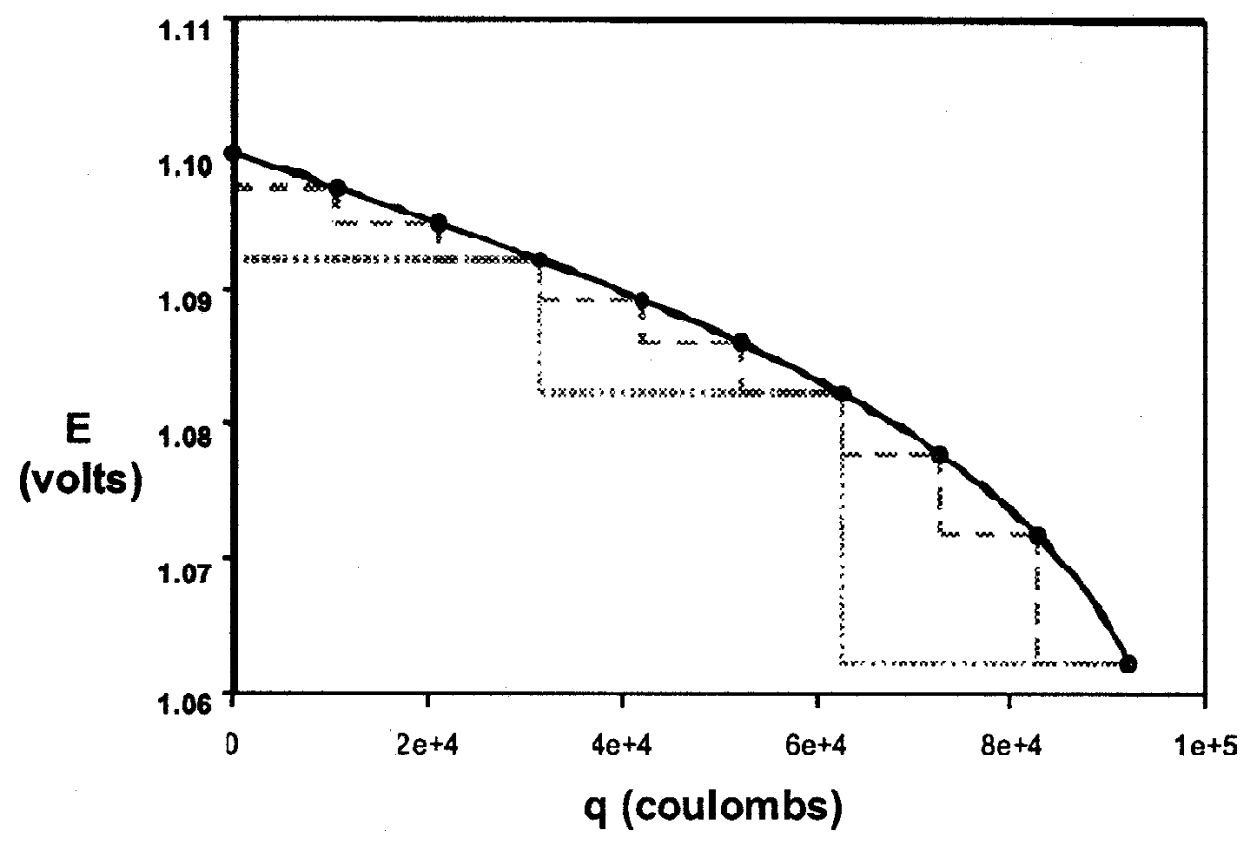


como demasiado complejos por los alumnos, tales como trabajo eléctrico y reversibilidad en un proceso diferente al que aplica la analogía. Simultáneamente, ha permitido reemplazar la imagen de celdas galvánicas y celdas electrolíticas ubicadas en compartimentos estancos por la de un proceso único. Además, esta estrategia generó una excelente oportunidad para una revisión integradora de los conceptos propios de la analogía.

Uno de los factores determinantes del éxito o fracaso de una analogía es el tiempo dedicado a la misma (Friedel, Gabel y Samuel, 1990). En este caso, la analogía es un tema más del programa de la materia desarrollado previamente con cierto detalle y reiterado posteriormente en su papel de analogía. De esta forma, el tiempo total dedicado a la comprensión de la analogía, previo su uso como puente con electroquímica, es suficiente para garantizar cierta eficacia. Simultáneamente tampoco se entorpece el desarrollo normal del curso como podría ocurrir si la analogía a utilizar fuera extracurricular y exigiera, sólo para su comprensión, una parte importante del tiempo dedicado al nuevo tema.

Debe indicarse que toda analogía, y ésta no es la excepción, tiene la posibilidad de ser extendida incorrectamente provocando desconceptos en el alumno. De este modo, interpretaciones «literales» de ciertos aspectos de la analogía o predicciones que la analogía prevé pero que no son directamente transferibles pueden provocar transposiciones del propio modelo acarreando errores con- ceptuales en los estudiantes debido a la dificultad de superar la propia analogía. Es evidente que, más allá de las similitudes entre analogía y hecho a explicar, existen claras diferencias entre ambos (muchas de las cuales se originan en que la analogía corresponde a un proceso físico mientras que en las celdas electroquímicas está ocurriendo un proceso químico), que no deben pasar desapercibidas para el alumno. Es por eso que ninguna estrategia de introducir nuevos conceptos a través de analogías o modelos puede ser consistente y adecuada si no existe una instancia posterior en la cual, ya incorporados los nuevos conceptos en el esquema cognitivo del alumno, se discuta en profundidad, similitudes y diferencias entre el nuevo tema incorporado y la analogía que sirvió de puente para su interpretación y comprensión.

Resumiendo, este trabajo ha tratado de mostrar que una analogía que funciona como puente entre un proceso más intuitivo y sencillo como el de expansión-comprensión isotérmica de un gas ideal y otro generalmente menos accesible para los alumnos, como es el proceso electroquímico, permite explotar las similitudes significativas entre ambas y desarrollar una visión de una aplicación más amplia de conceptos ya incorporados. Por último, como continuación de esta estrategia de aprendizaje, este trabajo puede fomentar la generación espontánea de otras analogías adecuadas mediante una discusión profunda entre el docente y los alumnos de otras similitudes y diferencias.

\section{REFERENCIAS BIBLIOGRÁFICAS}

ATKINS, P. y JONES, L. (1997). Chemistry. Molecules, Matter, and Change. Nueva York: W.H. Freeman and Company.

BROWN, T.L., LEMAY, H.E. y BURSTEN, B.E. (1998). Química: La ciencia central. Editorial Prentice Hall.

CHANG, R. (1998). Química. Madrid: McGraw-Hill.

DONATI, E.R. y ANDRADE GAMBOA, J.J. (1990). La utilidad de las analogías en la enseñanza de las ciencias en base a una posible clasificación. Enseñanza de las Ciencias, 8(1), pp. 89-91.

FRIEDEL, A.W., GABEL, D.L. y SAMUEL, J. (1990). Using Analogs for Chemistry Problem Solving: Does it Increase Understanding? School Science and Mathematics, 90(8), pp. 674-682.

GARNETT, P.J. y TREAGUST, D.F. (1992a). Conceptual Difficulties Experienced by Senior High School Students of Electrochemistry: Electric Circuits and Oxidation-Reduction Equations. Journal of Research in Science Teaching, (29)2, pp. 121-142.
GARNETT, P.J. y TREAGUST, D.F. (1992b). Conceptual Difficulties Experienced by Senior High School Students of Electrochemistry: Electrochemical (Galvanic) and Electrolytic Cells. Journal of Research in Science Teaching, 29(10), pp. 1079-1099.

POZO, J.I. y GÓMEZ CRESPO, M.A. (1998). Aprender y enseñar ciencia. Madrid: Morata.

POZO, J.I., SANZ, A., GÓMEZ CRESPO, M A. y LIMÓN, M. (1991). Las ideas de los alumnos sobre la ciencia: una interpretación desde la psicología cognitiva. Enseñanza de las Ciencias, 9(1), pp. 83-94.

SENGER, M.J. y GREENBOWE, T.J. (1997). Students' Misconceptions in Electrochemistry: Current Flow in Electrolyte Solutions and the Salt Bridge. Journal of Chemical Education,74(7), pp. 819-823.

WHITTEN, K.W., GAILEY, K.D. y DAVIS, R.E. (1992). Química general. Madrid: McGraw-Hill.

[Artículo recibido en junio de 2000 y aceptado en abril de 2001.] 